\title{
Profissionais que trabalham com AIDS e suas representações sociais sobre o atendimento e o tratamento
}

\author{
Social representations of professionals who \\ work with AIDS attendance and treatment
}

\author{
Cristiane Galvão RIBEIRO \\ Maria da Penha Lima COUTINHO² \\ Ana Alayde Werba SALDANHA \\ Alessandra Ramos CASTANHA
}

\begin{abstract}
Resumo
Esta pesquisa teve como objetivo identificar, nos profissionais que atendem pacientes soropositivos para o HIV, as representações sociais sobre $\mathrm{o}$ atendimento e $\mathrm{o}$ tratamento da doença. A amostra foi composta por 61 profissionais - em sua maioria, do sexo feminino (82\%), com idade superior a 44 anos (42\%) - que trabalham com a AIDS nos serviços de saúde pública de João Pessoa, PB. Os dados foram coletados pela técnica de associação livre de palavras, processados pelo software Tri-Deux-Mots e interpretados por meio da análise fatorial de correspondência, em cujo gráfico emergiram universos semânticos, que são agrupamentos de palavras, associados à idade e à categoria profissional, classificados na área médica e na área de humanas. Percebeu-se que a experiência leva o profissional a ultrapassar as questões do sofrimento que a AIDS provoca e a redirecionar o atendimento e tratamento aos aspectos mais subjetivos. Formações acadêmicas diferentes influenciam as representações da soropositividade, e tais representações estão relacionadas a suas práticas profissionais.
\end{abstract}

Palavras-chave: profissional de saúde; representações sociais; sindrome de imunodeficiência adquirida.

\begin{abstract}
This research has aimed the identification of AIDS attendance and treatment Social Representations on professionals that handle HIV serum-positive patients. The sample was composed by 61 professionals who work directly in the AIDS context at the Public Health from João Pessoa, PB. Most of them were women (82\%) and older than 44 years old (42\%). Data have been collected through the Word Free Association Technique, and later analyzed through Tri-Deux-Mots software, interpreted by the factorial correspondence analysis. The factorial correspondence analysis graphic revealed semantics universes associated with the age and professional categories, which was classified into the medical and humanities area. It was observed that the daily practice experience leads the professional to go beyond the suffering issues caused by AIDS, as well as to re-conduct the attendance and treatment towards more subjective aspects of the patient. Different academic background influences the serum-positive representations development, as it is related to the personal daily practices.
\end{abstract}

Key words: health personnal; social representations; acquired immunodeficiency syndrome.

1 Mestrandas, Programa de Pós-Graduação em Psicologia, Universidade Federal da Paraíba. João Pessoa, PB, Brasil.

2 Professoras Doutoras, Programa de Pós-Graduação em Psicologia, Centro de Ciências Humanas Letras e Artes, Universidade Federal da Paraíba. Cidade Universitária, Campus I, 58059-900, João Pessoa, PB, Brasil. Correspondência para/Correspondence to: C.G. RIBEIRO. E-mail: <cristianegr@ig.com.br>. 
A AIDS (síndrome da imunodeficiência adquirida) demonstra nitidamente a complexidade do fenômeno saúde/doença próprio da realidade contemporânea. Inicialmente, levava à morte num curto espaço de tempo, não havendo tratamento adequado. Em quinze anos, ou seja, até 1995, a AIDS levou a morte cerca de 70.337 pessoas no Brasil (Galvão 2001).

Em 1986, foi aprovada, nos Estados Unidos, a primeira droga anti-retroviral, a aziditimidina ou AZT, revelando um discreto impacto sobre a mortalidade dos pacientes. A partir de 1996, um novo conjunto de drogas passou a ser estudado, os inibidores da protease, que demonstrou, isoladamente ou em associação com as do grupo AZT (coquetel), potente efeito anti-retroviral, levando à diminuição da mortalidade imediata, à melhora dos indicadores da imunidade e à recuperação de infecções oportunistas (Aoki, 2001), e diminuindo drasticamente o número de óbitos por AIDS no mundo.

A partir de tal fato, a história da doença pode ser contada antes e depois da introdução do esquema combinado de anti-retrovirais, colocando a AIDS no rol das doenças crônicas. A eficácia da terapia antiretroviral de inibidores da protease é evidente, no entanto, a continuidade dessa trajetória não está livre de desafios. As principais dificuldades observadas pelos profissionais de saúde que lidam com a AIDS têm sido a não adesão dos pacientes ao tratamento. Segundo Jordan, Lopes, Okazaki, Komatsu e Nemes (2000), os serviços de saúde têm sido cada vez mais apontados como importantes determinantes da adesão para as doenças crônicas em geral.

A prática em saúde tem acompanhado a perspectiva vigente, acentuando, de certa forma, a discrepância entre o conhecimento científico e as demandas psicossociais exigidas pelo atendimento aos pacientes, requerendo dos profissionais uma postura mais voltada ao aspecto afetivo das relações, colocando-os frente a suas próprias limitações diante de uma realidade fragmentada.

A AIDS trouxe consigo a necessidade de reformular a estrutura no atendimento em saúde já estabelecida, impondo a necessidade de atenção ao paciente como um todo e não apenas a um órgão doente. Trouxe também a demanda de lidar com plano, fazendo emergir o despreparo e a desorientação que envolvem os profissionais de saúde no trato psicossocial da doença (Saldanha, 2003), enquanto o tratamento clínico é favorecido por constantes descobertas.

Dessa forma, a cronicidade da AIDS coloca os serviços de saúde, representados pelos profissionais que lidam diretamente com a doença, num patamar extremamente importante, não só para garantir a adesão dos pacientes, mas para que se tornem um elo entre o paciente, a doença e o tratamento.

Nesse sentido, torna-se relevante compreender a problemática que envolve os serviços de atendimento à AIDS. Essa tarefa se faz tão importante quanto os fenômenos de prevenção primária, contribuindo com o processo e exercício da cidadania no âmbito da saúde.

Diante do pressuposto, optou-se por utilizar o enfoque teórico das representações sociais (Moscovici, 1978), por permitir apreender o conhecimento baseado na experiência social comum, através da expressão dos atores sociais (profissionais que trabalham no contexto da AIDS). Segundo Jodelet (1998), a densidade existencial intrínseca à área da saúde e a especificidade da articulação entre o conhecimento científico e os saberes tradicionais favorecem a atualização de significações e de valores socialmente partilhados para a compreensão dos quais a abordagem das representações sociais é particularmente heurística.

As representações sociais, na medida em que orientam a organização da vivência corporal e das relações com a saúde, são tratadas como variáveis explicativas, tanto da defasagem constatada entre os dados epidemiológicos e o acesso ao sistema médico, quanto dos limites da eficácia das campanhas educativas de orientação das condutas de promoção da saúde, de prevenção das doenças e de adesão aos tratamentos (Jodelet, 1998).

Conforme Tura (1998), para uma estratégia de prevenção e intervenção ou criação de atitudes em relação à doença, devem-se considerar o complexo de emoções, elaborações mentais, teorias práticas e explicativas do cotidiano, que se inserem na constituição das representações sociais e atuam concretamente nas 
escolhas elaboradas pelos indivíduos diante da incerteza e ameaça que a AIDS provoca.

Partindo dessas premissas, este estudo objetivou apreender as representações sociais dos profissionais que trabalham diretamente no contexto da AIDS, acerca do seu atendimento e tratamento, bem como comparar as representações sociais desses profissionais advindos de formações acadêmicas das áreas de Saúde e de Humanas.

\section{Método}

\section{Participantes}

A amostra foi constituída por 61 profissionais que trabalham no contexto da AIDS, em três serviços de saúde pública que atendem pacientes HIV positivos, na cidade de João Pessoa, PB, sendo: 16 médicos, 16 enfermeiros, 12 psicólogos e 17 assistentes sociais, dos quais $18 \%$ são do sexo masculino e $82 \%$ do sexo feminino, com idade variando de 24 a 50 anos. É válido mencionar que a escolha das quatro categorias profissionais se deve ao fato de elas lidarem de forma direta com os pacientes HIV positivos em suas práticas cotidianas.

\section{Instrumentos}

Foram utilizados um questionário com o fim de obter dados sociodemográficos e a Técnica de Associação Livre de Palavras (Di Giácommo, 1984), que consiste em um tipo de investigação aberta que se estrutura na evocação de respostas com base em um ou mais estímulos indutores. Neste estudo foram utilizados, como estímulos indutores, as palavras: AIDS (1), atendimento (2) e tratamento (3).

\section{Procedimentos}

Inicialmente, foi mantido contato com a diretoria das instituições com a finalidade de obter autorização para coleta dos dados. Os instrumentos foram aplicados no ambiente de trabalho dos profissionais, durante o horário de plantão, de forma individual. Na oportunidade, explicitaram-se os objetivos da pesquisa e a garantia do anonimato e confidencialidade das respostas.
Após a coleta dos dados sociodemográficos, aplicou-se a técnica de associação livre de palavras, fazendo a cada participante a seguinte pergunta: "Se eu Ihe digo a palavra AIDS (primeiro estímulo indutor), quais as cinco primeiras palavras que Ihe vêm à mente?" O mesmo procedimento foi utilizado para o segundo e terceiro estímulo indutores. Cada participante levou, em média, um minuto para responder a cada estímulo.

Em seguida, foi preparado um dicionário de respostas a fim de categorizar os conteúdos emergentes para serem processados através do software Tri-Deux-Mots (Cibois, 1991) e interpretados por meio da análise fatorial de correspondência (AFC).

O uso da AFC é útil para explicar as representações sociais apreendidas no teste de associação livre de palavras, pois evidencia as variáveis fixas neste estudo (profissão, sexo e idade) em relação às variáveis de opiniões (palavras evocadas pelos participantes) que se confrontam e se revelam graficamente na representação do plano fatorial (Coutinho, 2001).

\section{Resultados}

Na Figura 1 estão apresentados os dados coletados pelo Teste de Associação Livre de Palavras acerca da representação social da AIDS, seu atendimento e tratamento, construídos com base nos estímulos indutores. A Figura 1 demonstra uma representação das variações semânticas na organização do campo espacial, ilustrando-se aproximações e oposições das modalidades na construção dos fatores (F1 e F2). Os dois fatores foram detentores de um poder explicativo de $71,9 \%$ da variância total de respostas, sendo que o F1 apresentou 42,2\% e o F2, 29,7\%.

No fator 1 (em itálico), no campo esquerdo, encontra-se o campo semântico das representações sociais da AIDS elaboradas pelos profissionais da área de ciências humanas. Para esse grupo, a AIDS (estímulo 1) é ancorada nas doenças sexualmente transmissíveis, e demanda apoio das pessoas que convivem com o paciente através do amor e da solidariedade. O atendimento (estímulo 2) para essa categoria se efetiva através do respeito e empatia ao paciente, e o tratamento 
(estímulo 3), segundo os atores sociais, exige orientação dos profissionais através da atenção e respeito.

Ainda no F1 (em itálico), no campo direito, encontram-se as representações sociais dos profissionais inseridos na faixa etária entre 24 e 33 anos, independentemente da área de atuação. Para esses profissionais a AIDS é uma doença associada à depressão, morte e sofrimento. $\mathrm{O}$ atendimento para esse grupo se resume à consulta, é considerado precário e acontece sob os critérios de objetividade e rapidez, embora percebam a demanda da solidariedade. O tratamento para esses profissionais é associado à necessidade de informação.

No fator 2 (em negrito), na parte superior, observou-se um campo semântico relacionado aos profissionais acima de 44 anos, independente da categoria profissional. Para eles, a AIDS é uma doença que demanda apoio das pessoas que lidam com o paciente. $O$ atendimento para a categoria se efetiva por meio do apoio e da compreensão e é preciso que o profissional oriente os pacientes

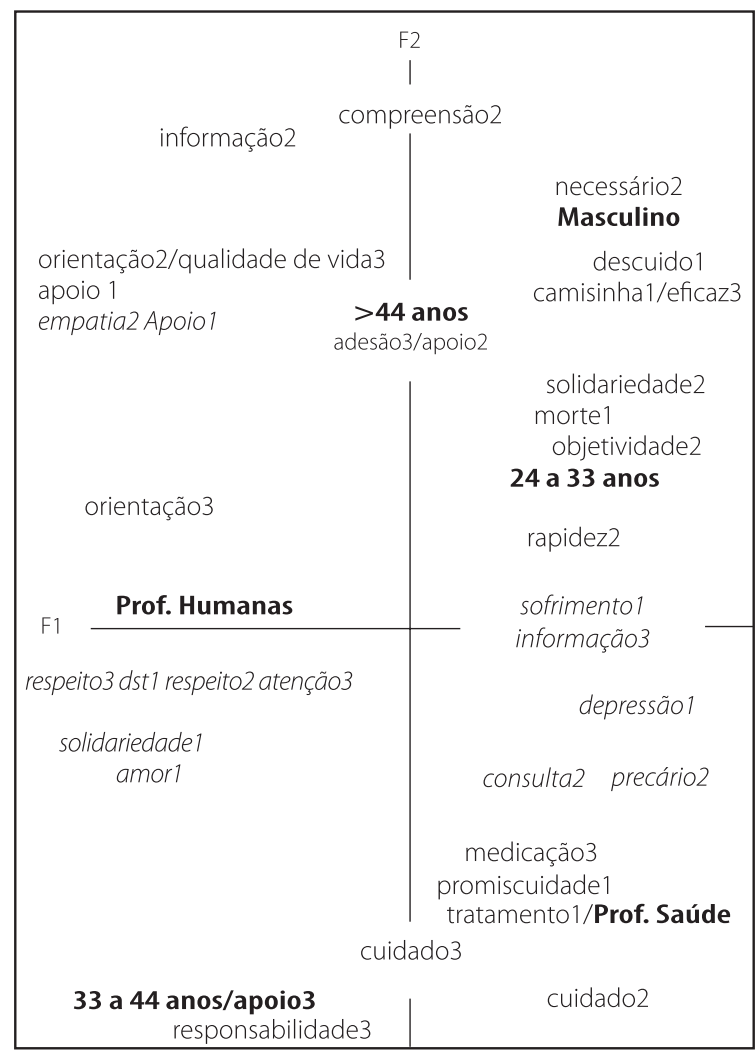

Figura 1. Representações sociais dos profissionais das áreas de Saúde e Humanas acerca da AIDS, seu atendimento e 78 tratamento. através da informação, para que haja adesão ao tratamento e, conseqüentemente, uma melhoria na qualidade de vida.

Ainda no fator 2 (em negrito), também na parte superior, emergiu um campo semântico associado à categoria dos profissionais do sexo masculino, que vêem a AIDS como uma doença proveniente do descuido pelo não uso da camisinha. $O$ atendimento para eles é considerado um serviço necessário e pode ser estabelecido através do apoio e da compreensão. O tratamento é percebido como eficaz, mas é preciso adesão por parte dos pacientes.

No fator 2 (em negrito), na parte inferior, emergiram as representações sociais dos profissionais da área médica. Para esse grupo a AIDS é uma doença originada da promiscuidade e que precisa de tratamento. $O$ atendimento para eles exige certo cuidado por parte dos profissionais em relação ao contágio/acidente de trabalho, ou seja, em relação ao risco de contaminação na prática com os pacientes. O tratamento se efetiva através da ingestão da medicação, a qual exige cuidado.

Ainda no fator 2 (em negrito) na parte inferior, observam-se as representações sociais de profissionais independentemente da categoria profissional em que estão inseridos na faixa etária dos 33 aos 44 anos, que associam o tratamento da AIDS à necessidade de apoio e cuidado, para que haja responsabilidade pelos pacientes.

Esses resultados expressam que as representações sociais sobre a AIDS e seu atendimento por profissionais diretamente envolvidos no trato com o paciente soropositivo para o HIV estruturam-se na interface da complexidade, colocando em jogo conhecimentos científicos e do senso comum, bem como valores sociais e contextuais.

\section{Discussão}

É preciso articular, nas análises, as concepções evocadas e as implicações psicossociais que thes fazem eco. Com relação à faixa etária, foram observadas, nas representações sociais dos profissionais com idade entre 24 e 33 anos, questões sobre o sofrimento provocado pela AIDS, ao associarem-na à morte e à depressão. 
Segundo Tura (1998), esse sofrimento se deve às incertezas e às ameaças diante da doença.

Tal fato confirma o enunciado de Malbergier e Stempliuk (1997) quando tratam a questão "AIDS/ morte". Para os autores, alguns profissionais relatam não se sentirem instrumentalizados para abordar o assunto com seus pacientes, principalmente aqueles com menos experiência, uma vez que nos cursos de graduação não há treinamento para abordar a questão. Ensina-se a combater ou retardar a morte, mas não se ensina a discuti-la, remetendo o profissional à sua própria limitação e levando-o às questões sobre a própria vida e identidade profissional.

Segundo Jodelet (1998), a associação entre AIDS e morte não é sistemática, mas se correlaciona significativamente com o tipo de relação mantida com os pacientes. Nesse sentido a distância favorece a visão de uma doença dos tempos modernos, enquanto relações de proximidade fazem prevalecer a visão de uma doença que mata. Diante dessa premissa, acredita-se que a aproximação cotidiana em relação à AIDS, vivenciada por esses profissionais, justifica a elaboração de representações da AIDS associadas à morte, evidenciando os achados de Jodelet (1998).

Em relação ao atendimento, a categoria dos profissionais com idade entre 24 e 33 anos valoriza questões associadas à praticidade no trato com os pacientes, não se referindo aos seus aspectos subjetivos. Segundo Figueiredo (2000), a racionalidade na prática em saúde desvitaliza o atendimento, já que a formação profissional orientada por essa lógica prioriza a competência técnica em detrimento da sensibilidade social. A rapidez e a objetividade, priorizadas por esse grupo, confirmam as informações advindas do Programa de Doenças Sexualmente Transmissíveis/AIDS de São Paulo, que preconiza que as consultas devam durar no mínimo 30 minutos, mas é comum durarem menos de quinze (Paiva, Leme, Nigro \& Caraciolo, 2000).

Com a prática cotidiana, o profissional parece redirecionar o foco do atendimento às questões mais específicas de cada paciente, respeitando suas singularidades, como exemplificado nos resultados, ao comparar as faixas etárias. Segundo Saldanha (2003), alguns estudos realizados pelo Programa de Atendimento Psicossocial à AIDS-(PAPSI) demonstram que a formação deve ser reorientada para a capacitação do profissional a fim de aproveitar essa aprendizagem informal, acumulada pela experiência no trato com o paciente.

Além disso, com a experiência adquirida, os profissionais demonstram conseguir ultrapassar as questões do sofrimento causado pela AIDS. Não que eles se tornem insensíveis, mas os anos vividos lado a lado com a doença ensinam a lidar melhor com a soropositividade.

Diante do exposto, observou-se que os profissionais mais jovens, através dos elementos figurativos morte, sofrimento e depressão, ilustram suas limitações frente à realidade que a AIDS proporciona, provavelmente pela pouca experiência. Verificaram-se também alguns elementos distintos e/ou opostos, como a objetividade, rapidez e precariedade do atendimento (profissionais com idade entre 24 e 33 anos) contrapondo-se à compreensão, apoio e informação (profissionais com idade acima de 44 anos). Tais distinções demonstram a probabilidade de haver mudanças nas representações desses profissionais em decorrência da experiência profissional.

Vale ressaltar que a informação e a orientação emergiram na maioria dos grupos como elemento importante tanto na dinâmica do atendimento quanto do tratamento. Segundo Melchior (2000), um dos fatores que dificultam a adesão ao tratamento são as dificuldades no estabelecimento da comunicação entre profissionais e pacientes, pois a superação dessas dificuldades passa pela possibilidade de informação e negociação com a equipe de saúde. Ainda sob o mesmo enfoque, Saldanha (2003) relata que a falta de informação sobre a doença e o tratamento leva o paciente a construir, com seus próprios meios, um discurso com materiais fragmentados. Nesse sentido, a informação e a orientação surgem como poderosos recursos utilizados por esses profissionais, tanto no estabelecimento da qualidade do atendimento, como, conseqüentemente, na adesão ao tratamento.

Em relação à categoria profissional, como esperado, foi observado que as representações dos profissionais de humanas ultrapassam os aspectos físicos, demonstrando uma maior preocupação com a subjetividade do atendimento. Nesse sentido, Alves e Ramos (2002) ressaltam que o profissional aperfeiçoa-se quando percebe que uma doença 
nunca é igual para duas pessoas, e suas causas e conseqüências dependem em grande parte do perfil psicológico, meio em que vive e da situação econômica e social do paciente.

Entretanto, em algumas instituições de referência ao HIV, percebe-se, na prática profissional de psicólogos e assistentes sociais, a priorização das entrevistas de coleta de dados dos pacientes em detrimento de uma atitude de verdadeira escuta. Nesse sentido surge a necessidade de um contato maior que ultrapasse os aspectos formais, levando profissional e paciente ao estabelecimento de um vínculo satisfatório.

Ainda sob esse enfoque, Saldanha (2003) relata a observação de um grande comprometimento dos profissionais com seu trabalho e compromisso com o paciente, no entanto, tal postura não pode ser confundida com uma atitude de verdadeira escuta, com a priorização das informações do paciente. Saldanha enfoca o primeiro contato profissional/ paciente, por meio do preenchimento de fichas de anamnese, transformando a atitude de escuta em coleta de dados sobre o paciente.

Quanto às representações sociais dos profissionais da área médica, percebe-se a presença de elementos ancorados nas primeiras representações, associadas à promiscuidade. Essa maneira de encarar a doença é susceptível ao aparecimento do preconceito direcionado a grupos denominados marginais. Sob esse enfoque, Alves e Ramos (2002) relatam que em relação às doenças que trazem em si o preconceito e a discriminação social, a AIDS traz consigo um estigma maior, pois fala de sexo, sangue, homossexualismo, prostituição, traição, trazendo à tona o mais íntimo das pessoas, escondido através de tabus. Se não forem abordadas essas dificuldades, elas podem interferir no atendimento, gerando desconfiança, hostilidade, angústia e até abandono do tratamento (Malbergier \& Stempliuk 1997).

Para o referido grupo, o tratamento efetiva-se pelo uso da medicação, corroborando os achados de Silva, Oliveira, Figueiredo, Landroni, Waldman e Ayres, (2002), que ressaltam que o tratamento medicamentoso, sob a forma dos medicamentos anti-retrovirais, ocupa um lugar de destaque dentre as necessidades de saúde dos usuários dos centros de referência, sendo sua adesão um dos aspectos mais importantes, que suscita uma integração de esforços dos profissionais.

\section{Considerações Finais}

Com referência às categorias profissionais, emergiram semelhanças e diferenças entre as áreas estudadas. Ambas as categorias ancoraram a AIDS em doenças advindas de práticas sexuais (DST e promiscuidade), mas os profissionais da área das ciências humanas demonstraram um maior interesse nas demandas psicossociais dos indivíduos portadores do HIV. Os profissionais da área médica expressaram uma maior preocupação com questões fisiológicas da AIDS ao compararem-no a uma doença que necessita de tratamento, que se efetiva por meio da medicação.

Ainda em relação à ancoragem, além de associarem a AIDS a doenças sexualmente transmissíveis, percebeu-se um novo olhar desses profissionais, também voltado aos aspectos psicoafetivos, trazendo a depressão como elemento figurativo associado à doença, conforme percebido nos atores sociais inseridos na faixa etária entre 24 e 33 anos.

Observou-se que formações acadêmicas diferentes influenciam as formas de representar a questão da soropositividade, para as quais as representações sociais de cada grupo convergem com suas práticas cotidianas. Tal enunciado ilustra a predominância da informação técnica nos profissionais da área médica, contrapondo-se aos interesses psicossociais dos profissionais da área de humanas. É importante ressaltar que, se a informação técnica é essencial, também se faz necessária a habilidade para lidar com aspectos subjetivos para que haja a superação dos limites da compartimentalização do conhecimento.

Têm sido observadas mudanças favoráveis na atitude de profissionais no decorrer da epidemia de AIDS, mas o atendimento ao paciente permanece como um problema a ser discutido. Diante do exposto, muitos obstáculos surgem nos serviços que atendem o paciente soropositivo para o HIV, especificamente no que tange ao contato direto. O estigma trazido pela AIDS, aliado ao medo e sobrecarga profissional, faz 
emergir uma realidade precária nos serviços de saúde coletiva que dão assistência à AIDS.

Nesse sentido, as construções e partilhas específicas em cada grupo de pertença no atendimento e tratamento clínico da AIDS representaram um caminho para um universo consensual que não se restringiu aos limites da doença, mas, ao contrário, perpassaram aspectos técnicos, fazendo emergir representações que moldam as práticas cotidianas.

A partir dessas elucidações, pretende-se, além de compreender o fenômeno do atendimento à AIDS no Brasil, contribuir para um aperfeiçoamento dessas práticas cotidianas, tanto para promover uma melhoria na qualidade de vida dos atores sociais inseridos nesse contexto (profissionai s e pacientes) quanto para garantir o exercício da cidadania no âmbito da saúde pública.

\section{Referências}

Alves, E. G. R., \& Ramos, D. L. P (2002). Profissionais de saúde: vivendo e convivendo com HIV/AIDS. São Paulo: Santos.

Aoki, F. H. (2001). Leito-Dia em AIDS: uma experiência multiprofissional. In M. R. C Colombrini et al. (Orgs.). AIDS: epidemia e evolução do tratamento. São Paulo: Atheneu.

Cibois, P. H. (1991). L'analyse factorielle (3.ed). Paris: Puf. Collecion Que sais-je?.

Coutinho, M. P. L. (2001). Depressão infantil: uma abordagem psicossocial. João Pessoa: Editora Universitária.

Di Giacomo, J. C. (1984). Alliance et rejets untergroupes au seun d'um mouvement de revendication. In W. Doise et al. L'etude des representacions socials. Paris: Delchaux \& Niestle.

Figueiredo, M. A. C. (2000). AIDS, ciência e sociedade: a dicotomia entre conhecimento técnico e competência social no trabalho do profissional de saúde. In M. L. Boarini (Org.). Desafios na atenção à saúde mental. São Paulo: Eduem.
Galvão, J. (2001). 1980-2001: uma cronologia da epidemia de HIV/AIDS no Brasil e no Mundo. Rio de Janeiro: ABIA.

Jodelet, D. (1998). Representações sociais do contágio e a AIDS. In Jodelet. AIDS e Representações Sociais à busca de sentidos. Natal: EDUFRN.

Jordan, M.S., Lopes J. F, Okazaki E., Komatsu C. L, \& Nemes, M. I. B. (2000) Aderência ao Tratamento Anti-retroviral em AIDS: Revisão da Literatura Médica. In R. P. Teixeira \& V. Paiva. Tá difícil de engolir? Experiências de adesão ao tratamewnto anti-retroviral em São Paulo. São Paulo: Nepaids.

Malbergier, A., Stempliuk, V. A. (1997). Os médicos diante do paciente com AIDS: atitudes, preconceitos e dificuldades. Jornal Brasileiro de Psiquiatria, 46 (5).

Melchior, R. (2000). Avaliação da aderência de usuários do sistema público de assistência de assistência ao tratamento de AIDS: uma análise qualitativa. Dissertação de mestrado não-publicada, Departamento de Epidemiologia, Faculdade de Saúde Pública, Universidade de São Paulo.

Moscovici, S. (1978). A Representação social da Psicanálise. Rio de Janeiro: Zahar.

Paiva, V., Leme, B., Nigro, R., Caraciolo, J. (2000). Lidando com a adesão: A experiência de profissionais e ativistas na cidade de São Paulo. In R.P. Teixeira, V. Paiva (2000). Tá difícil de engolir? Experiências de adesão ao tratamento anti-retroviral em São Paulo. São Paulo: Nepaids.

Saldanha, A. A. W. (2003).Vulnerabilidade e construções de enfrentamento da soro positividade ao HIV por mulheres infectadas em relacionamento estável. Tese de doutorado não-publicada, Programa de Pós-Graduação em Psicologia, Faculdade de Filosofia, Ciências e Letras de Ribeirão Preto.

Silva, N. E. K, Oliveira, L. A., Figueiredo, W. S., Landroni, M. A. S., Waldman C. C. S., \& Ayres, J. R. C. M. (2002). Limites do trabalho multiprofissional: estudo de caso dos centros de referência para DST/AIDS. Revista de Saúde Pública, (4 supl): 108-116.

Tura, L. F. R.(1998). AIDS e estudantes: a estrutura das representações sociais. In D. Jodelet. AIDS e representações sociais à busca de sentidos. Natal: Edufrn.

Recebido para publicação em: 22/4/2005

Versão final reapresentada em: 11/5/2005

Aprovado em: 29/9/2005 